\title{
Temporal profiles of blood pressure, circulating nitric oxide, and adrenomedullin as predictors of clinical outcome in acute ischemic stroke patients
}

\author{
MARTA SERRANO-PONZ ${ }^{1}$, CARMEN RODRIGO-GASQUÉ ${ }^{2}$, EVA SILES $^{3}$, ESTHER MARTÍNEZ-LARA $^{3}$, \\ LAURA OCHOA-CALLEJERO ${ }^{4}$ and ALFREDO MARTÍNEZ ${ }^{4}$
}

\begin{abstract}
${ }^{1}$ Stroke Unit, Neurology Service; ${ }^{2}$ Radiology Unit, Hospital San Pedro, 26006 Logroño; ${ }^{3}$ Experimental Biology Department, University of Jaén, 23071 Jaén; ${ }^{4}$ Angiogenesis Group, Oncology Area, Center for Biomedical Research of La Rioja (CIBIR), 26006 Logroño, Spain
\end{abstract}

Received December 3, 2015; Accepted February 18, 2016

DOI: $10.3892 / \mathrm{mmr} .2016 .5001$

\begin{abstract}
Stroke remains an important health and social challenge. The present study investigated whether blood pressure (BP) parameters and circulating levels of nitric oxide metabolites (NOx) and adrenomedullin (AM) may predict clinical outcomes of stroke. Patients $(n=76)$ diagnosed with acute ischemic stroke were admitted to the stroke unit and clinical history data and monitored parameters were recorded. Blood plasma was collected at days 1, 2, and 7 to measure NOx and AM levels. Infarct volume, neurological severity [on the National Institutes of Health Stroke Scale (NIHSS)], and functional prognosis (on the Rankin scale) were measured as clinical outcomes. Patients with higher BP had more severe symptoms (NIHSS $>3 ; \mathrm{P}<0.01$ ) and $\mathrm{BP}$ variability predicted neurological severity and growth of infarct volume. NOx values were significantly lower in stroke patients than in healthy controls $(\mathrm{P}<0.01)$. An increase in NOx levels from day 1 to day 2 was beneficial for the patients as measured by NIHSS at 7 days and 3 months, and by Rankin at 3 months [odds ratio (OR), 0.91] whereas a steep increase from day 2 to day 7 was detrimental and associated with an increase in infarct volume (OR, 35.3). AM levels were significantly higher in patients at day 1 and 2 than in healthy
\end{abstract}

Correspondence to: Professor Alfredo Martínez, Angiogenesis Group, Oncology Area, Center for Biomedical Research of La Rioja (CIBIR), 98 Piqueras, 26006 Logroño, Spain

E-mail: amartinezr@riojasalud.es

Abbreviations: AM, adrenomedullin; $\mathrm{BP}$, blood pressure; $\mathrm{CV}$, coefficient of variation; DAP, diastolic blood pressure; MRI, magnetic resonance imaging; NO, nitric oxide; NOS, NO synthase; NOx, nitrate/nitrite and S-nitroso compounds; ROC, receiving operating characteristic; SAP, systolic arterial pressure; SD, standard deviation; $\mathrm{SV}$, successive variation

Key words: adrenomedullin, blood pressure variability, ischemic stroke, nitric oxide, temporal profiles individuals $(\mathrm{P}<0.01)$ and these levels returned to normal at day 7. Patients with high AM levels at day 2 had significantly higher NIHSS scores measured at day $1(\mathrm{P}<0.05)$ and 7 $(\mathrm{P}<0.01)$. A receiving operating characteristic curve analysis identified that AM levels at day 2 of $>522.13 \mathrm{pg} / \mathrm{ml}$ predicted increased neurological severity at day 7 (area under the curve $=0.721$ ). Multivariate logistic regression indicated that AM levels at day 2 predicted increased neurological severity at 7 days and at 3 months. BP parameters and changing levels for NOx and AM predicted long-term clinical outcomes as measured by infarct volume, neurological severity scale, and functional prognosis.

\section{Introduction}

A stroke or cerebrovascular accident occurs when an area of the brain is suddenly deprived of blood flow. This may be due to the occlusion of a blood vessel (ischemic stroke) accounting for $\sim 80 \%$ of all strokes, or to a local intracranial hemorrhage (hemorrhagic stroke). The lack of nutrients and oxygen alters the metabolism of the affected neurons and glial cells and results in the appearance of neurological symptoms and signs that may become irreversible, depending on the time prior to circulation being re-established $(1,2)$. Stroke constitutes a important health and social problem; according to the World Health Organization, $\sim 15$ million people suffer a stroke every year and, of these, 5 million are fatal and 5 million result in permanent incapacitation (3). Recent data suggests that up to $85 \%$ of all strokes may be preventable using medical intervention and lifestyle modifications (4), however, urgent care is required at emergency stroke units.

A search for biomarkers that may predict clinical outcomes in stroke patients is ongoing $(5,6)$. As stroke is a vascular disease, vasoactive substances are particularly notable. Thus, the present study investigated nitric oxide (NO) and adrenomedullin (AM). NO is a gaseous free radical that is synthesized from L-arginine by nitric oxide synthases (NOS). NO may exert opposite functions in the central nervous system depending on its concentration, site of production, and the NOS isoform that produces it. Low concentrations 
produced by endothelial NOS are often beneficial and contribute to local vasodilatation. However, large concentrations of NO, such as those produced by the inducible NOS isoform result in the excessive generation of free radicals and the destruction of biological molecules, including proteins, lipids, and nucleic acids (7-9). It has been demonstrated that NO production increases under hypoxia (10), a common situation in tissues affected by stroke. In periods of ischemia, $\mathrm{NO}$ production is reduced due to oxygen deficiency, however, immediately following reperfusion, synthesis of this molecule is triggered by activation of neuronal and endothelial NOS. A number of hours later, NO concentration increases again due to the activation of the inducible NOS isoform (11). Thus, modulators of NO may provide neuroprotection following neurovascular accidents. NO donors markedly reduce infarction volume in experimental models $(12,13)$. Recently, circulating NO levels have been proposed as a biomarker to predict mortality in ischemic stroke patients (14). However, as NO is a free radical with a short half life, only its chemical products, nitrate/nitrite and S-nitroso compounds (NOx) are measurable in clinical samples (7).

$\mathrm{AM}$ is a vasodilatatory peptide produced by numerous areas of the central nervous system (15) and peripheral tissues (16). As with NO, AM expression is also upregulated under hypoxia via activation of the hypoxia inducible factor-1 pathway (17) and it has been demonstrated to increase following ischemic insults to the brain (18-20). Previous studies in mice lacking AM expression suggest that this peptide is neuroprotective in the context of stroke, and may exert its effects via regulation of inducible NOS, matrix metalloproteinases, and inflammatory mediators (21).

The present study aimed to perform a longitudinal follow-up of stroke patients measuring blood pressure, NO, and AM levels and investigating whether these values may be predictive of clinical parameters, including infarct volume growth, neurological severity, or functional prognosis.

\section{Materials and methods}

Ethical issues. All procedures were approved by the local review board (Comité Ético de Investigación Clínica de La Rioja, Logroño, Spain) and all described procedures adhere to the tenets of the Declaration of Helsinki.

Patients. The present study was designed as a prospective, observational, and longitudinal clinical study with patients diagnosed with acute ischemic stroke at the Neurology Service of the Hospital San Pedro (Logroño, Spain) from October 2014 to April 2015. Consecutive patients $(n=76)$ fulfilling inclusion criteria signed the written informed consent documents and were recruited into the current study. Inclusion criteria required patients to be suffering from acute ischemic stroke demonstrated by magnetic resonance imaging (MRI), and an evolution of $<24 \mathrm{~h}$. Exclusion criteria were the same used for the hospital's stroke unit and were as follows: Contraindications for performing MRI; age, <18 years; dementia; previous stroke within 3 months; cranial traumatism; central nervous system infection; cardiac insufficiency; renal failure; sepsis; active neoplasia; active inflammatory or autoimmune disease; pregnant or lactating women; and patients who would not be able to complete proper follow-up.

In this time period, 140 patients were diagnosed and treated at the stroke unit. Of these, 127 were suspected of suffering ischemic stroke and 120 arrived within $24 \mathrm{~h}$ of the first symptoms. A total of 29 patients were excluded from the present study due to fulfilling at least one of the exclusion criteria and 15 additional patients were excluded following performing MRI as the ischemic lesion could not be confirmed. Finally, 76 patients were included in the study and all required data was collected for 70 of them.

Variables of the present study. Patients received standard care following the approved protocols of the stroke unit. General characteristics of the patient were collected as part of the clinical history (including age, gender, risk factors, current medical treatment and previous functional situation). During their stay at the stroke unit, numerous parameters were continuously monitored, including electrocardiogram, systolic arterial pressure (SAP) and diastolic arterial pressure (DAP), temperature, and hypoxemia. Neurological severity was measured with the National Institutes of Health Stroke Scale (NIHSS) (22) at 0, 24 h, 7 days, and 3 months. Functional prognosis was also evaluated with the Rankin scale at 3 months (23). In addition, blood plasma samples were taken on day 1 , day 2 , and day 7 to quantify circulating levels of NO and AM. Infarct volume evolution was established by comparing the images captured by MRI on day 1 and at day 7 with a 3 Tesla instrument (Discovery MR 750w; GE Healthcare Life Sciences, Chalfont, UK).

Determination of NOx levels. NO production was indirectly quantified by determining NOx, using an ozone chemiluminescence-based assay adapted to plasma samples (24). Briefly, plasma samples were deproteinized with $0.8 \mathrm{~N} \mathrm{NaOH}$ and $16 \% \mathrm{ZnSO}_{4}$ solutions (1/0.5/0.5, v/v/v; Sigma-Aldrich, Madrid, Spain). Following centrifugation at $10,000 \mathrm{x} \mathrm{g}$ for $5 \mathrm{~min}$ at room temperature, the resulting supernatants were removed for chemiluminescence analysis in a $\mathrm{NO}$ analyzer $\left(\mathrm{NOA}^{\mathrm{TM}} 280 \mathrm{i}\right.$; GE Analytical Instruments, Boulder, CO). NOx concentration was calculated by comparison with standard solutions of sodium nitrate. Final NOx values were expressed in $\mu \mathrm{M}$.

Determination of AM levels. The concentration of AM present in blood plasma was determined using a commercially available radioimmunoassay (RIA) kit (Phoenix Europe $\mathrm{GmbH}$, Karlsruhe, Germany). Samples (1 ml) were initially diluted in an equal volume of $0.1 \%$ alkali-treated casein in phosphate-buffered saline at $\mathrm{pH}$ 7.4, and applied to pre-washed reverse-phase Sep-Pak C-18 cartridges (Waters Corporation, Milford, MA, USA) to remove the AM-binding protein, complement factor $\mathrm{H}$. The peptide fraction was eluted from the C-18 matrix with $3 \mathrm{ml}$ $80 \%$ isopropanol containing $0.125 \mathrm{~N} \mathrm{HCl}$ and freeze-dried overnight, as previously described (25). AM levels contained in lyophilized extracts were then determined by RIA following the manufacturer's protocols.

Statistical analysis. All statistical analyses were performed using the SPSS version 21 software package (IBM SPSS, Armonk, NY, USA). A descriptive analysis of all variables 
Table I. Clinical characteristics of the 76 patients included in the present study.

Clinical characteristic

Total

Age, mean \pm SD

$73.31 \pm 12.33$

Gender (M)

$43(56.6 \%)$

Risk factors

Arterial hypertension

$53(69.7 \%)$

Diabetes mellitus

$24(31.6 \%)$

Dyslipidemia

$38(50 \%)$

Ischemic cardiopathy

$9(11.8 \%)$

Atrial fibrillation

$16(21,1 \%)$

Previous stroke

$14(18.5 \%)$

Previous treatment

Antiagreggants

$25(32.9 \%)$

Anticoagulants

$10(13.2 \%)$

Antihypertensives

$54(71.1 \%)$

Statins

$30(39.5 \%)$

Previous Rankin

$0-1$

$67(88.2 \%)$

2

$6(7.9 \%)$

$3(3.9 \%)$

3

Basal NIHSS, median (Q1-Q3)

$36.1 \pm 0.4$

Temperature at ER $\left({ }^{\circ} \mathrm{C}\right)$, mean $\pm \mathrm{SD}$

$124.9 \pm 37.4$

Glycemia at ER $(\mathrm{mg} / \mathrm{dl})$, mean $\pm \mathrm{SD}$

$160.1 \pm 30.0$

$\mathrm{SAP}$ at ER $(\mathrm{mm} \mathrm{Hg})$, mean $\pm \mathrm{SD}$

$84.8 \pm 18.7$

TOAST

Atherothrombotic

$25(32.9 \%)$

Cardioembolism

$27(35.5 \%)$

Lacunar

$11(14.5 \%)$

Other

Basal infarct volume $\left(\mathrm{cm}^{3}\right)$, median (Q1-Q3)

$3.8(0.9-17.2)$

Time from symptoms to MRI (min), median (Q1-Q3)

$960(540-1260)$

Basal AM (pg/ml), median (Q1-Q3)

$488.7(411.5-631.6)$

Basal NOx $(\mu \mathrm{M})$, median (Q1-Q3)

$9.26(6.88-14.34)$

SD, standard deviation; NIHSS, National Institutes of Health Stroke Scale; ER, emergency room; SAP, systolic blood pressure; DAP, diastolic blood pressure; TOAST, Trial of Org 10172 in Acute Stroke Treatment; MRI, magnetic resonance imaging; AM, adrenomedullin ; NOx, nitric oxide metabolites.

was performed and categorical variables were expressed as absolute and relative frequencies. Continuous variables were defined by their mean and standard deviation (SD) when their distribution was normal (as tested by the Shapiro-Wilk test) or as the median and interquartile range when the distribution was not normal. Univariate analyses were performed with Pearson's $\chi^{2}$ test, modified by Fisher's exact test, or in the case of continuous variables with Student's t-test or analysis of variance. When samples did not follow a normal distribution, non-parametric tests, including Kruskal Wallis followed by Mann-Whitney's U test were performed. Parameters that were marked as significant in univariate analysis entered multivariate binary logistic regression to investigate independent effects. A receiving operating characteristic (ROC) analysis was performed to assess the potential predictive value of binary classifier systems. $\mathrm{P}<0.05$ was considered to indicate a statistically significant difference.

\section{Results}

Clinical characteristics of patients. The clinical sample included 76 stroke patients, 33 women (43.4\%) and 43 men $(56.6 \%)$, with a mean age of $73.3 \pm 12.3$ years (Table I). Age-matched healthy controls were 5 women (35.7\%) and 9 men $(64.3 \%)$ with a mean age of $73.4 \pm 12.2$ years. Certain patients had been exposed to relevant risk factors, including arterial hypertension, dyslipidemia, diabetes, atrial fibrillation, ischemic cardiopathy, or a previous stroke (Table I). A 
A

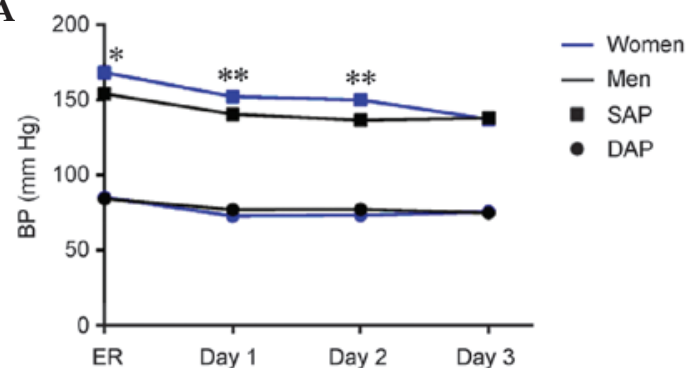

C

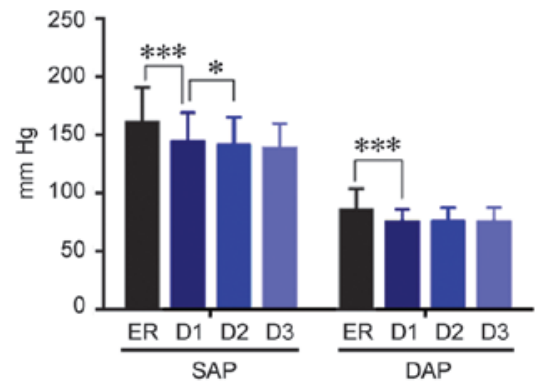

B

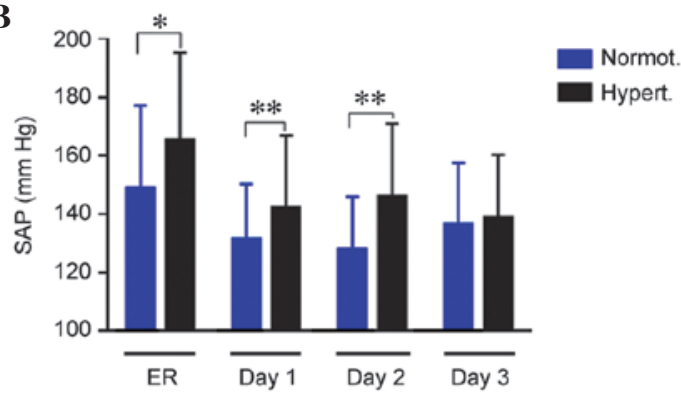

D

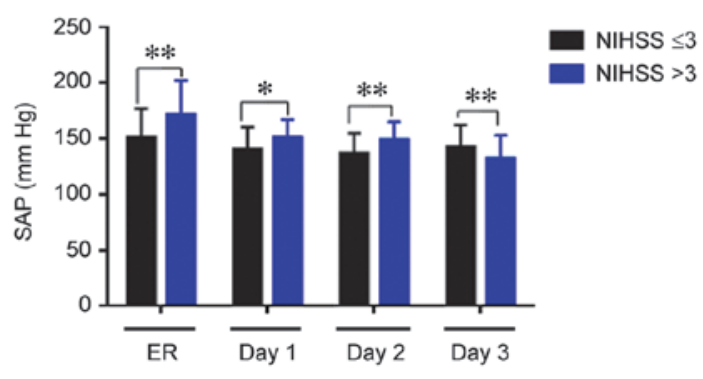

Figure 1. Evolution of BP parameters during the stay at the stroke unit and association with the NIHSS. SAP and DAP were measured at the ER and during the next 3 days. (A) Women had significantly higher SAP than men. ${ }^{*} \mathrm{P}<0.05$ and ${ }^{* *} \mathrm{P}<0.01$ vs. the data from male patients. (B) At admission and at day 1 and 2 , SAP was higher in patients with a history of hypertension. (C) SAP and DAP were reduced over time due to the stroke unit care. (D) There was a direct association between higher SAP and higher neurological severity measured at $24 \mathrm{~h}$. Bars represent the mean \pm standard deviation. ${ }^{*} \mathrm{P}<0.05 ;{ }^{* *} \mathrm{P}<0.01{ }^{* * * *} \mathrm{P}<0.001$. BP, blood pressure; NIHSS, National Institutes of Health Stroke Scale; SAP, systolic arterial pressure; DAP, diastolic arterial pressure; ER, emergency room; D1, day 1; D2, day 2; D3, day 3.

marked proportion of these patients had been treated with antiaggregants, anticoagulants, antihypertensives, and statins (Table I). Following completion of the etiological profile, $\sim 1 / 3$ of the patients were diagnosed with atherothrombotic stroke, another third with cardioembolic stroke, and $14.5 \%$ with lacunar stroke (Table I). The median NIHSS taken at admission was 3 , with a median infarct volume of $3.8 \mathrm{~cm}^{3}$ (Table I). Intracranial or extracranial occlusion of a large vessel was demonstrated in 17 patients (22.3\%).

Blood pressure variability was associated with neurological severity and infarct volume growth. At admission in the emergency room (ER), mean values for SAP and DAP were $160.1 \pm 30.0 \mathrm{mmHg}$ and $84.8 \pm 18.7 \mathrm{mmHg}$, respectively (Table I). Hypertension was observed at the admission of $59(77.6 \%)$ patients. Women exhibited significantly higher SAP values than men at admission $(\mathrm{P}=0.04)$, and on day 1 $(\mathrm{P}=0.008)$ and day $2(\mathrm{P}=0.003)$ of treatment; however, this difference was corrected by day 3 . No significant differences by gender were observed for DAP (Fig. 1A). Patients with a history of hypertension exhibited significantly higher SAP values, as compared with normotensive patients at admission $(\mathrm{P}=0.03)$, and on days $1(\mathrm{P}=0.004)$ and $2(\mathrm{P}=0.002)$ of treatment (Fig. 1B). DAP values were similar independently of hypertension history. There was a significant reduction in SAP and DAP $(\mathrm{P}<0.001)$, during the first day of treatment, and a further reduction in SAP $(\mathrm{P}<0.05)$ from day 1 to day 2 (Fig. 1C). When comparing blood pressure and neurological severity measured by the NIHSS at $24 \mathrm{~h}$, patients with more severe symptoms (NIHSS $>3$ ) had significantly higher SAP (Fig. 1D; ER, $\mathrm{P}=0.002$; day $1, \mathrm{P}=0.014$; and day $2, \mathrm{P}=0.005$ ) and DAP (data not shown) until day 2, with this trend reversing at day 3 (Fig. $1 \mathrm{D} ; \mathrm{P}=0.009)$, as compared with patients with less severe symptoms (NIHSS $\leq 3$ ).

Variability in blood pressure (BP) values has been proposed as a possible cause of brain deterioration in ischemic stroke patients (26). This variability is established by measuring the SD, the successive variation, and the coefficient of variation of either the SAP or the DAP. Notably, almost all variability parameters were significantly higher in women when compared with men (Table II). This higher variability was also observed in patients with increased neurological severity as measured by the NIHSS at $24 \mathrm{~h}$ and at 7 days (Table II). In addition, patients whose infarct volume grew during the first 7 days had significantly higher $\mathrm{BP}$ variability than those whose infarct volume did not grow (Table II). There was no association between the variability in BP during the stay of the patients in the stroke unit and their performance at 3 months, measured by either the NIHSS or the Rankin scale (data not shown).

NOx levels were significantly lower in stroke patients compared with healthy controls at day 1 and 2. In healthy control subjects, NOx levels were $15.3 \mu \mathrm{M}$ (12.1-17.9). These levels were significantly higher in women than in men $(\mathrm{P}=0.02$; data not shown). In stroke patients, NOx levels measured on day 1 and day 2 of their hospital stay were significantly lower $(\mathrm{P}=0.008)$ than those obtained in healthy subjects. At day 7, NOx levels were significantly higher than at day $1(\mathrm{P}<0.001)$ and day $2(\mathrm{P}<0.01)$, but indistinguishable from healthy controls (Fig. 2A). In stroke patients, no differences were observed with gender. 
Table II. Variability of BP compared with gender, NIHSS at $24 \mathrm{~h}$ and 7 days, and with infarct volume growth.

A, BP variability vs. gender

\begin{tabular}{lcrr}
\hline Variability & Men & Women & P \\
\hline SAP-SD & $12.5(10.1-14.56)$ & $14.6(12.3-18.9)$ & $\mathbf{0 . 0 0 3}$ \\
SAP-SV & $13.1(11.9-15.3)$ & $17.2(12.9-20)$ & $\mathbf{0 . 0 2 0}$ \\
SAP-CV & $9.3(7.4-11.1)$ & $9.8(7.9-13.8)$ & 0.090 \\
DAP-SD & $8.4(7.3-10)$ & $12.5(10-15.8)$ & $\mathbf{0 . 0 3 0}$ \\
DAP-SV & $10.9(8.5-13)$ & $14.3(10.7-17.8)$ & $\mathbf{0 . 0 3 0}$ \\
DAP-CV & $9.2(6.7-14.4)$ & $\mathbf{0 . 0 0 3}$ \\
\hline
\end{tabular}

$\mathrm{B}, \mathrm{BP}$ variability vs. NIHSS at $24 \mathrm{~h}$

\begin{tabular}{lccc}
\hline Variability & NIHSS $\leq 3$ & NIHSS $>3$ & P \\
\hline SAP-SD & $12.5(10.4-15.4)$ & $14.4(12-19.3)$ & $\mathbf{0 . 0 0 1}$ \\
SAP-SV & $13.3(10.4-15.4)$ & $16.7(13-20.9)$ & $\mathbf{0 . 0 0 1}$ \\
SAP-CV & $9.1(7.2-11.2)$ & $9.9(8-13.7)$ & 0.200 \\
DAP-SD & $8.3(7.2-10.5)$ & $9.8(8.4-12.7)$ & $\mathbf{0 . 0 1 4}$ \\
DAP-SV & $9.7(8-12.4)$ & $12(10.7-15.6)$ & $\mathbf{0 . 0 0 3}$ \\
DAP-CV & $11.1(7-14.5)$ & $12.6(8.1-16.2)$ & 0.210 \\
\hline
\end{tabular}

C, BP variability vs. NIHSS at 7 days

\begin{tabular}{lccc}
\hline Variability & NIHSS $\leq 3$ & NIHSS $>3$ & P \\
\hline SAP-SD & $12.6(9.9-16)$ & $13.7(12.5-19.4)$ & $\mathbf{0 . 0 2 0}$ \\
SAP-SV & $13.1(10.8-18)$ & $15.6(13.3-22)$ & $\mathbf{0 . 0 1 2}$ \\
SAP-CV & $9.3(7.2-11.8)$ & $9.9(8.4-12)$ & 0.300 \\
DAP-SD & $8.4(7.3-10.6)$ & $12.5(9.9-16)$ & $\mathbf{0 . 0 1 7}$ \\
DAP-SV & $10.6(8.2-12)$ & $12.6(8.1-16.2)$ & $\mathbf{0 . 0 1 3}$ \\
DAP-CV & $11.7(7.8-14.4)$ & & 0.360 \\
\hline
\end{tabular}

D, BP variability vs. infarct volume growth

\begin{tabular}{lccr}
\hline Variability & No growth & Growth & P \\
\hline SAP-SD & $12.6(10.4-15.8)$ & $14.2(12.3-21.1)$ & $\mathbf{0 . 0 2 0}$ \\
SAP-SV & $13.8(12.3-16.8)$ & $16.3(12.3-22.9)$ & 0.100 \\
SAP-CV & $8.9(7.3-10.7)$ & $10.7(9-14)$ & $\mathbf{0 . 0 2 0}$ \\
DAP-SD & $8.3(7.5-9.9)$ & $11(8.5-12.6)$ & $\mathbf{0 . 0 0 6}$ \\
DAP-SV & $10.6(8.5-12)$ & $13.3(10.4-16.3)$ & $\mathbf{0 . 0 0 4}$ \\
DAP-CV & $11.2(6.9-14.3)$ & $14.2(9-17.6)$ & 0.050 \\
\hline
\end{tabular}

Statistically significant P-values are represented in bold. BP, blood pressure; NIHSS, National Institutes of Health Stroke Scale; SAP, systolic arterial pressure; DAP, diastolic arterial pressure; SD, standard deviation; SV, successive variation; CV, coefficient of variation.

When NOx levels were compared with neurological severity, it was observed that patients with an NIHSS $\leq 3$ at day 1 had significantly higher values of $\mathrm{NOx}$ on day 2 (Fig. $2 \mathrm{~B} ; \mathrm{P}=0.02$ ). There was also an association with BP parameters. Patients in the third tertile of NOx levels at day 1 had a significantly lower DAP on days $2(\mathrm{P}=0.009)$ and $3(\mathrm{P}=0.02$; Fig. $2 \mathrm{C})$. Patients in the third tertile of SAP at admission had significantly lower
$(\mathrm{P}=0.01) \mathrm{NOx}$ levels than other patients in the second and first tertile (Fig. 2D).

AM levels were significantly higher than in healthy controls at day 1 and 2. Healthy control volunteers had a median AM value of $389.7 \mathrm{pg} / \mathrm{ml}$ (343.9-475.9). No differences were observed for either gender or age groups. In stroke patients, AM levels 
A

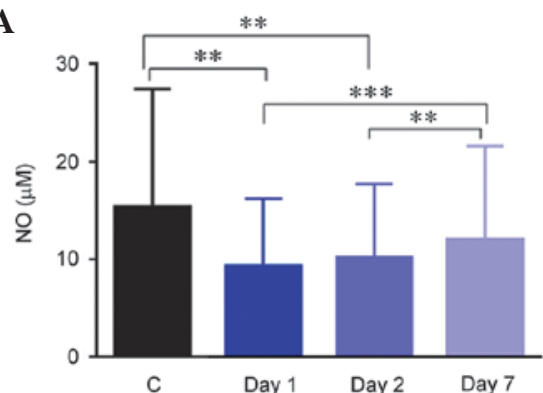

C

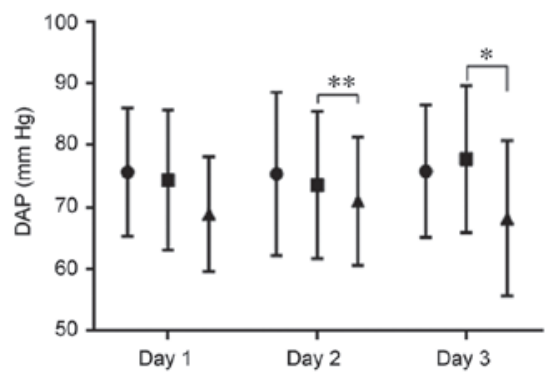

B

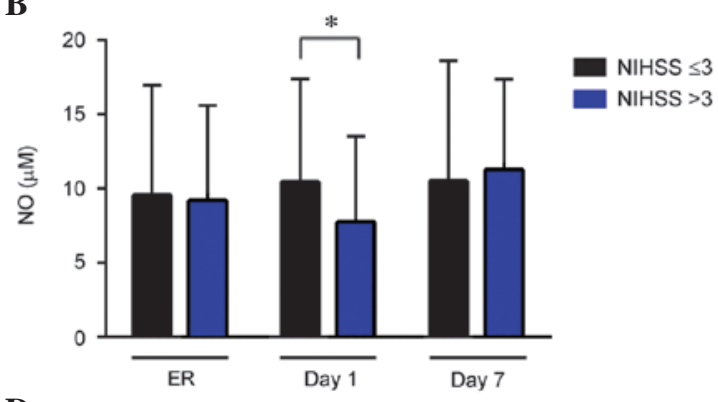

D

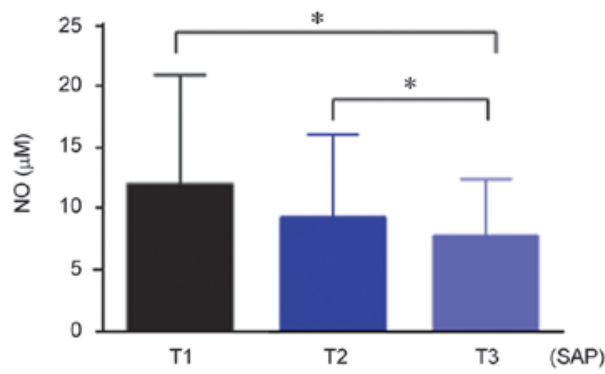

Figure 2. Evolution of NOx levels and their association with NIHSS and blood pressure. (A) The amount of circulating NOx was measured in healthy controls and stroke patients at days 1,2, and 7. (B) Patients with higher NOx levels measured at day 2 had a lower NIHSS score at day 1. (C) When NOx data were divided in tertiles, patients in the third (highest) tertile had lower DAP at day 2 and 3. (D) Also, patients with higher SAP (third tertile) at admission had the lowest levels of circulating NOx. Bars represent the mean \pm standard deviation for DAP and the median \pm interquartile range for $\mathrm{NO}$. $\mathrm{P}<0.05$; ${ }^{* *} \mathrm{P}<0.01$; ${ }^{* * * *} \mathrm{P}<0.001$. NIHSS, National Institutes of Health Stroke Scale; NO, nitric oxide; NOx, nitrate/nitrite and S-nitroso compounds; DAP, diastolic arterial pressure; SAP, systolic arterial pressure; C, control; T1, tertile 1; T2, tertile 2; T3, tertile 3.

A

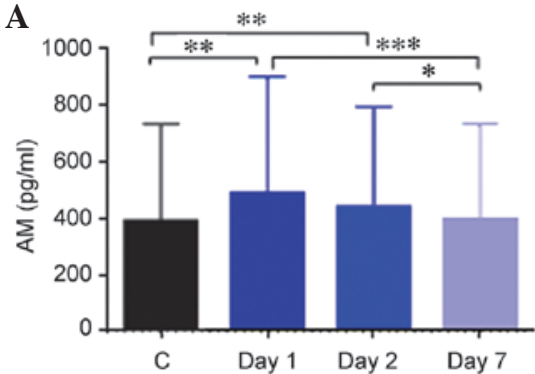

C

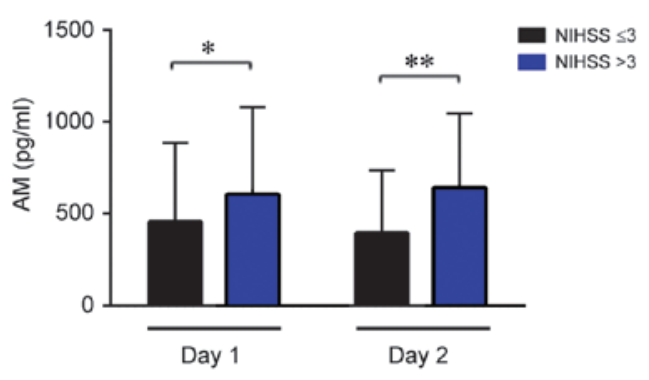

B

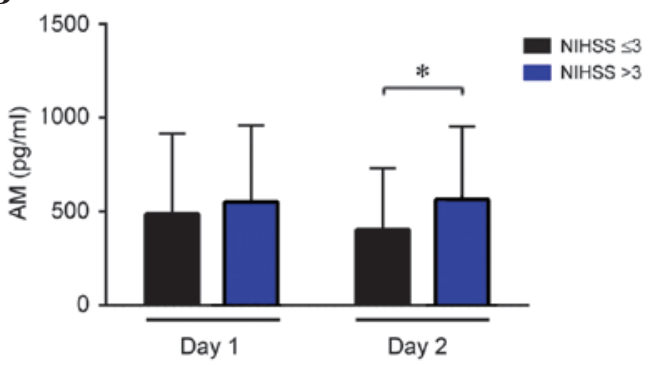

D

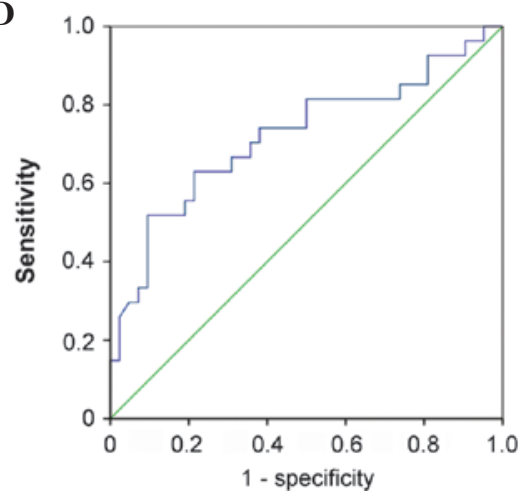

Figure 3. Evolution of AM levels and their association with NIHSS. (A) The amount of circulating AM was measured in healthy controls and stroke patients at days 1, 2, and 7. Patients with higher AM levels had a higher NIHSS score measured either at (B) $24 \mathrm{~h}$ or at (C) 7 days. (D) A receiving operating characteristic analysis for AM predicted NIHSS at 7 days with an area under the curve of 0.721 , a sensitivity of $70 \%$, and a specificity of $60 \%$. Bars represent the median \pm interquartile range. ${ }^{*} \mathrm{P}<0.05 ;{ }^{* *} \mathrm{P}<0.01 ;{ }^{* * *} \mathrm{P}<0.001 . \mathrm{AM}$, adrenomedullin; NIHSS, National Institutes of Health Stroke Scale.

were significantly higher than in healthy controls at day 1 $(\mathrm{P}=0.003)$ and $2(\mathrm{P}=0.005)$, however, these values were indistinguishable from controls at day 7 (Fig. 3A). Among patients, women had significantly higher AM levels than men
$(\mathrm{P}=0.02$; data not shown). Notably, patients that had undergone treatment with antiaggregants or statins previous to the stroke had significantly lower levels of AM as measured at day $2(\mathrm{P}=0.04$ and $\mathrm{P}=0.001$, respectively; data not shown). 
Table III. Univariate analysis of potential predictors of infarct volume growth.

\begin{tabular}{|c|c|c|c|}
\hline \multirow[b]{2}{*}{ Parameter } & \multicolumn{2}{|c|}{ Growth } & \multirow[b]{2}{*}{ P-value } \\
\hline & No & Yes & \\
\hline All patients $(\mathrm{n}=70)$ & $44(62.9 \%)$ & $26(37.1 \%)$ & \\
\hline Gender (men) & $28(71.8 \%)$ & $11(28.2 \%)$ & 0.080 \\
\hline Age & $74.79 \pm 10,13$ & $70.92 \pm 14.71$ & 0.170 \\
\hline Hypertension & $29(61.7 \%)$ & $18(38.3 \%)$ & 0.700 \\
\hline Diabetes & $16(76.2 \%)$ & $5(23.8 \%)$ & 0.100 \\
\hline Dyslipidemia & $23(67.6 \%)$ & $11(32.4 \%)$ & 0.410 \\
\hline Ischemic cardiopathy & $6(75.0 \%)$ & $2(25.0 \%)$ & 0.430 \\
\hline Atrial fibrillation & $8(47.1 \%)$ & $9(52.9 \%)$ & 0.410 \\
\hline Previous stroke & $10(76.9 \%)$ & $3(23.1 \%)$ & 0.220 \\
\hline \multicolumn{4}{|l|}{ Previous treatments } \\
\hline Antihypertensives & $34(70.8 \%)$ & $14(29.2 \%)$ & 0.040 \\
\hline Statins & $20(71.4 \%)$ & $8(28.6 \%)$ & 0.200 \\
\hline Antiaggregants & $19(82.6 \%)$ & $5(14.4 \%)$ & 0.010 \\
\hline Anticoagulants & $5(55.6 \%)$ & $4(44.4 \%)$ & 0.600 \\
\hline Basal NIHSS & $2(1-5)$ & $6(2-11)$ & 0.007 \\
\hline 24 h NIHSS & $1(0-3)$ & $4(1-5)$ & 0.003 \\
\hline Basal glycemia (mg/dl) & $105.18 \pm 38.15$ & $110.42 \pm 29.34$ & 0.050 \\
\hline 72 h glycemia & $116.05 \pm 34.12$ & $113.06 \pm 26.33$ & 0.620 \\
\hline Hyperthermia & $7(43.8 \%)$ & $9(56.3 \%)$ & 0.070 \\
\hline SAP at ER & $157.2 \pm 25.5$ & $167 \pm 36.0$ & 0.200 \\
\hline DAP at ER & $81.3 \pm 15.2$ & $91.5 \pm 22.3$ & 0.046 \\
\hline TOAST & & & 0.710 \\
\hline Atherothrombotic & $17(70.8 \%)$ & $7(29.2 \%)$ & \\
\hline Cardioembolic & $15(62.5 \%)$ & $9(34.5 \%)$ & \\
\hline Lacunar & $5(50.0 \%)$ & $5(50.0 \%)$ & \\
\hline Other strokes & $7(58.3 \%)$ & $5(41.7 \%)$ & \\
\hline Large vessel occlusion & $5(35.7 \%)$ & $9(64.3 \%)$ & $\mathbf{0 . 0 2 0}$ \\
\hline Basal infarct vol. $\left(\mathrm{cm}^{3}\right)$ & $2.8(0.6-0.5)$ & $3.9(1.1-28.2)$ & 0.050 \\
\hline Day 1 NOx $(\mu \mathrm{M})$ & $9.3(8-14.6)$ & $9.1(5.9-14.4)$ & 0.300 \\
\hline Day 2 NOx & $10.6(7.6-13.6)$ & $9.9(6.9-14)$ & 0.300 \\
\hline NOx increase (day 7-day 1) & $5(1.9-12.2)$ & $1.2(0-7.6)$ & 0.030 \\
\hline Day 1 AM (pg/ml) & $497.8(442-647.4)$ & $565.9(403.5-637.6)$ & 0.610 \\
\hline Day 2 AM & $428.5(355.6-571.4)$ & $440.8(352.6-708.3)$ & 0.520 \\
\hline AM increase (day 7-day 1) & $-125.6(-216.8-(-) 34.9)$ & $-151.9(-228.6-(-) 30)$ & 0.700 \\
\hline
\end{tabular}

Statistically significant P-values are represented in bold font. NIHSS, National Institutes of Health Stroke Scale; SAP, systolic arterial pressure; ER, emergency room; DAP, diastolic arterial pressure; TOAST, Trial of Org 10172 in Acute Stroke Treatment; NOx, nitrate/nitrite and S-nitroso compounds; AM, adrenomedullin; SD, standard deviation; SV, successive variation; CV, coefficient of variation.

Patients with a history of hypertension had similar levels of AM to the other stroke patients, however, their AM concentration decline from day 1 to 7 was significantly steeper than in other patients $(\mathrm{P}=0.005$; data not shown $)$.

There was no association between AM levels and neurological severity as measured using the NIHSS at admission. However, patients with a high NIHSS score ( $>3)$ at day 1 had significantly higher AM levels at day 2 (Fig. 3B; $\mathrm{P}=0.014$ ). A similar association was observed with NIHSS values measured at day 7 and AM values obtained on day 1 and day 2 of treatment (Fig. 3C; $\mathrm{P}=0.012$ and $\mathrm{P}=0.002$, respectively).
These data suggested that AM levels measured on day 2 may predict neurological severity at day 7 . To investigate this hypothesis, a ROC curve analysis was performed and it was demonstrated that the optimal threshold for AM levels was $522.13 \mathrm{pg} / \mathrm{ml}$. This value renders an area under the curve of 0.721 (95\% confidence interval, 0.590-0.852), a sensitivity of $70 \%$, and a specificity of $60 \%$ (Fig. 3D).

Previous treatment with antihypertensives and antiaggregants was protective against infarct volume growth. Infarct volume growth was defined as the difference between the infarct volume 
Table IV. Multiparametric analysis (multivariate logistic regression) identifying independent predictors for each of the clinically meaningful parameters.

\begin{tabular}{lccr}
\hline Parameter & OR & $95 \%$ CI & P-value \\
\hline Infarct volume growth & & & 0.030 \\
Antiaggregants & 0.12 & $0.019-0.799$ & 0.040 \\
24 h NIHSS & 7.50 & $1.01-56.07$ & 0.030 \\
Large vessel occlusion & 9.36 & $1.23-71.11$ & 0.006 \\
NOx increase (day 7-day 2) & 35.3 & $2.8-439.6$ & 0.002 \\
Neurological severity (NIHSS) at 7 days & & & 0.040 \\
Gender (male) & 0.02 & $0.85-0.99$ & 0.020 \\
NOx increase (day 2-day 1) & 0.92 & $1.45-44.7$ & 0.010 \\
AM (day 2) & 8.07 & $1.62-43.1$ & 0.001 \\
24 h NIHSS & 8.37 & $5.38-120.38$ & 0.030 \\
Large vessel occlusion & 80.43 & & 0.030 \\
Neurological severity (NIHSS) at 3 months & & $0.82-0.99$ & 0.020 \\
NOx increase (day 2-day 1) & 0.90 & $1.14-20.83$ & 0.030 \\
24 h NIHSS & 4.90 & $1.25-23.94$ & 0.040 \\
AM increase (day 2-day 1) & 5.46 & & $0.017-0.59$ \\
Functional prognosis (Rankin) at 3 months & & $0.84-0.99$ & \\
Gender (male) & 0.10 & 0.91 & \\
NOx increase (day 2-day 1) & & \\
\hline
\end{tabular}

OR, odds ratio; CI, confidence interval; NIHSS, National Institutes of Health Stroke Scale; NOx, nitrate/nitrite and S-nitroso compounds; AM, adrenomedullin.

measured by MRI at day 7 and the initial volume measured on day 1 of treatment. Patients were classified as having infarct volume growth when they exhibited a $\geq 20 \%$ increase, 26 patients (37.1\%) experienced such growth while 44 (62.9\%) did not. Demographic, clinical, and analytical characteristics of the patients were examined to investigate whether any had predictive value over the potential infarct volume growth as measured at day 7 (Table III). Notably, among the reported risk factors, only a previous treatment with antihypertensives or antiaggregants resulted in a clear protection against infarct volume growth $(\mathrm{P}=0.04$ and $\mathrm{P}=0.01$, respectively). There was a linear association between NIHSS scores $(>3)$, either at admission or at $24 \mathrm{~h}$, and volume growth. There was also an association with elevated diastolic blood pressure but not with the systolic component. In addition, patients with occlusion of a large vessel were more likely to experience infarct growth $(\mathrm{P}=0.02)$. The association with $\mathrm{NOx}$ was notable as there was no association with specific NOx levels but with the increment in $\mathrm{NOx}$ concentration from day 1 to $7(\mathrm{P}=0.03)$. No association was observed with AM levels.

Multiparametric analysis predicted male gender and increase in NOx levels from day 1 to day 2 improved prognosis. Subsequent to conducting univariate analysis, multivariate logistic regressions were performed to identify independent predictors of clinical outcomes. These outcomes were the growth of the infarct volume (day 7 - day 1), the NIHSS scores measured at day 7 and at 3 months after the onset of the condition, and the Rankin scale, also measured at 3 months (Table IV). As predictors of infarct volume growth it was demonstrated that pretreatment with antiaggregant therapeutic agents protects patients, whereas a high NIHSS score taken at $24 \mathrm{~h}$, the confirmation of the occlusion of a large vessel, and the steep increase on NOx levels provide a poorer prognosis. For neurological severity measured at day 7 , it was observed that male gender and the increase in NOx levels from day 1 to day 2 are protective, whereas a high NIHSS score taken at $24 \mathrm{~h}$, confirmation of the occlusion of a large vessel, and the increase of AM levels from day 1 to day 2 are markers of a poorer prognosis. For the neurological severity measured at 3 months the increase in NOx levels from day 1 to day 2 is a positive marker for improved recovery, whereas a high NIHSS score at $24 \mathrm{~h}$ and increased AM levels are negative predictors. As measured by functional prognosis at 3 months, male gender and a high increase in NOx levels from day 1 to day 2 are reliable predictors for a good prognosis (Table IV).

\section{Discussion}

The present study has identified that different parameters associated with blood pressure and circulating levels of NOx and AM may act as predictors of clinical outcome in acute ischemic stroke patients.

Previous treatments and infarct volume growth. It was observed in the present study that patients that had been treated with either antihypertensive or platelet antiaggregant therapeutic agents prior to stroke onset were protected against the growth of the infarct volume. It has been demonstrated that in-hospital treatment with these therapeutic agents may 
explain the decrease in mortality from ischemic stroke (27), and that taking these therapeutic agents prior to stroke onset may have a marked positive prognosis $(28,29)$. Data from the present study confirms these previous observations.

$B P$. In the patients investigated in the present study, women had a higher SAP than men at admission. This gender bias has been widely reported, despite men and women having similar risk factors (30). This difference between genders and the commonly high values in BP were progressively controlled during day 1 and 2 of stay in the stroke unit. The success of stroke units is a consequence of the control of all physiological factors that may influence the evolution of the ischemic lesion, referred to as non-pharmacological neuroprotection (31), which includes monitoring BP. Patients with higher BP presented higher values of neurological severity measured at $24 \mathrm{~h}$, suggesting that lowering BP may be a good strategy to reduce brain injury. However, the scientific literature is divided on this issue. The majority of observational studies associate high BP with poor clinical outcomes $(32,33)$. By contrast, other reports show improved clinical evolution with higher BP levels (34), and low values of BP have been also associated with a poor prognosis and higher mortality (35). A previous study has suggested that a U-shaped association may be present, with high $\mathrm{BP}$ values inducing brain edema and hemorrhagic transformation and low BP values contributing to the transformation of the penumbra into infarcted area (36). Previous interventional studies using antihypertensive therapeutic agents suggest that reducing BP during the acute phase is safe and may reduce mortality at 3 months $(37,38)$, whereas other previous studies have demonstrated either no benefit (39) or a small increase in early adverse events (40). A recent review, which combines data from 26 articles and 17,011 patients, concluded that there is no evidence that reducing BP during the acute phase of the stroke may save lives or reduce disability (41).

However, the variability of DAP and SAP values during the acute phase may provide more information. In the present study, an increased BP variability was observed in women, in patients with higher NIHSS scores, and in patients where infarct volume growth was reported. This is in agreement with previous reports where BP variability has been associated with poor clinical outcome $(26,42,43)$.

NOx. The present study has demonstrated that peripheral concentrations of NOx in stroke patients are lower than in the healthy control population. Previous studies disagree on whether peripheral NOx increases or decreases following stroke. Certain previous studies agree with the results from the present study indicating lower NOx levels in patients $(44,45)$, whereas other studies have described elevated levels compared with healthy controls $(46,47)$. This discrepancy may be due to different methods of measuring NOx or to variations in the L-arginine pathway, as a result of endothelial dysfunction, leading to elevated levels of symmetric and asymmetric dimethylarginine. These metabolites are elevated in stroke patients and result in reduced production of NO (48). Depending on the quantity of these metabolites, the final levels of NOx in the blood may vary. In addition, the potential contributions of NOS-independent sources of NO must be considered (49). In the patients investigated in the current study, during the first week of the follow-up period, NOx levels returned to normal, suggesting a progressive recovery of homeostasis. This elevation of NOx levels post-stroke is in agreement with previous studies (14).

The present study demonstrated that elevations of NOx levels may be beneficial or detrimental for the patient, depending on when they occur. Elevation of NOx from day 1 to day 2 was demonstrated to be protective and it predicted a positive outcome at 7 days and 3 months, for neurological recovery and functional dexterity. However, an elevation of NOx from day 2 to day 7 predicted growth of the infarct volume. This dual behavior may be associated with the type of NOS isoform that is activated. The initial elevation of NOx production may be associated with the endothelial NOS, an isoform that has been demonstrated to be neuroprotective via exerting an effect on vasodilatation, inhibition of platelet aggregation, and induction of angiogenesis (50). The elevation occurring from day 2 to 7 may be more associated with the activation of the inducible NOS isoform, the activity of which is initiated at $\sim 12 \mathrm{~h}$ after ischemic onset and continues for up to 8 days later (51). This isoform is considered damaging to the surrounding tissue due to the unregulated large quantities of NO produced (7).

As expected, an inverse association between NOx levels and BP was observed, confirming the vasodilatatory effect of NO in a clinical setting, as has been thoroughly reported in experimental models $(7,9)$.

$A M$. The present study observed that stroke patients had significantly higher values of plasma AM than healthy individuals, this is in agreement with previous literature $(20,52)$. By day 7, AM levels returned to normal, suggesting a progressive resolution of the pathology. A high AM level at day 2 or an increase in AM levels from day 1 to day 2 was associated with increased neurological severity at day 7 and at 3 months after the stroke. This was confirmed with a ROC curve analysis, where high AM values predicted a poorer outcome. A previous study has demonstrated that the AM level in the blood of ischemic stroke patients may be also predictive of 3-month mortality and unfavorable outcomes (52), suggesting $\mathrm{AM}$ is a negative predictor of clinical performance.

In experimental animals, it has been identified that eliminating AM from the central nervous system results in larger infarcts following acute ischemic stroke (21) and that injecting AM reduces infarct volume (53). These studies indicated the neuroprotective effects of AM are due to its vasodilatatory, pro-angiogenic, and anti-apoptotic effects. However, another previous study indicated intracerebroventricular injection of AM resulted in an increase of ischemic injury (54). This detrimental effect of AM may be mediated by its modulatory action on the immune system (55), which is important for stroke resolution (56). Overexpression of AM has been detected in leukocytes of stroke patients and this higher expression is associated with stroke severity (57). The clinical observations suggest that, during human stroke, AM levels may be a consequence of stroke severity, despite the neuroprotective effects of AM on the infarcted tissue.

The current study observed that patients taking antiaggregants or statins prior to stroke onset had lower levels of AM measured at day 2. Statins are well known for reducing cholesterol levels, however, they also exert pleiotropic actions, including antioxidative and cellular protective effects. These 
characteristics suggest that statins may provide novel therapeutic approaches for various neurological disorders, including stroke (58). There is little information regarding the association between AM and statins. Using AM heterozygous knockout mice, Yamamoto et al (59) demonstrated that statins significantly inhibited fibrosis and apoptosis while inducing angiogenesis in a model of heart fibrosis. The underlying mechanism for the protective action of statins in stroke may include a reduction of AM levels.

In conclusion, BP variability and temporal profiles of NOx and AM levels have been demonstrated as predictors of clinical outcomes in stroke patients, as measured by infarct volume growth, neurological NIHSS scales at 7 days and 3 months, and functional prognosis at 3 months. Development of rapid tests for evaluating NO and AM levels may be useful for predicting patient outcome, for developing personalized therapeutic strategies, and for stratifying stroke patients in clinical trials.

\section{Acknowledgements}

The authors would like to thank Dr. Enrique Ramalle-Gómara (Epidemiology Health Prevention Service, Logroño, Spain) for his help with the statistical analysis, Dr. Gemma Quincoces (Nuclear Medicine, University of Navarra, Pamplona, Spain) for her help with the $\gamma$-counter, and Ms. Judit Narro (Center for Biomedical Research of La Rioja, Logroño, Spain) for her excellent technical assistance. The present study was financed by Fundación Rioja Salud.

\section{References}

1. Albers GW, Caplan LR, Easton JD, Fayad PB, Mohr JP, Saver JL and Sherman DG; TIA Working Group: Transient ischemic attack-proposal for a new definition. N Engl J Med 347: 1713-1716, 2002.

2. Albers GW: Acute cerebrovascular syndrome: Time for new terminology for acute brain ischemia. Nat Clin Pract Cardiovasc Med 3: 521, 2006.

3. Mendis S, Davis S and Norrving B: Organizational update: The world health organization global status report on noncommunicable diseases 2014; one more landmark step in the combat against stroke and vascular disease. Stroke 46: e121-e122, 2015.

4. Sarikaya H, Ferro J and Arnold M: Stroke prevention-medical and lifestyle measures. Eur Neurol 73: 150-157, 2015.

5. Jickling GC and Sharp FR: Biomarker panels in ischemic stroke. Stroke 46: 915-920, 2015.

6. Wang Z, Lin Y, Liu Y, Chen Y, Wang B, Li C, Yan S, Wang Y and Zhao W: Serum uric acid levels and outcomes after acute ischemic stroke. Mol Neurobiol, 2015 (Epub ahead of print).

7. Rodrigo J, Fernández AP, Serrano J, Peinado MA and Martínez A: The role of free radicals in cerebral hypoxia and ischemia. Free Radic Biol Med 39: 26-50, 2005.

8. Terpolilli NA, Moskowitz MA and Plesnila N: Nitric oxide: Considerations for the treatment of ischemic stroke. J Cereb Blood Flow Metab 32: 1332-1346, 2012.

9. Garry PS, Ezra M, Rowland MJ, Westbrook J and Pattinson KT: The role of the nitric oxide pathway in brain injury and its treatment-from bench to bedside. Exp Neurol 263: 235-243, 2015.

10. Ho JJ, Man HS and Marsden PA: Nitric oxide signaling in hypoxia. J Mol Med (Berl) 90: 217-231, 2012.

11. Ito Y, Ohkubo T, Asano Y, Hattori K, Shimazu T, Yamazato M, Nagoya $\mathrm{H}$, Kato Y and Araki N: Nitric oxide production during cerebral ischemia and reperfusion in eNOS- and nNOS-knockout mice. Curr Neurovasc Res 7: 23-31, 2010.

12. Martínez-Murillo R, Fernández AP, Serrano J, Rodrigo J, Salas E, Mourelle M and Martínez A: The nitric oxide donor LA 419 decreases brain damage in a focal ischemia model. Neurosci Lett 415: 149-153, 2007.
13. Willmot M, Gray L, Gibson C, Murphy S and Bath PM: A systematic review of nitric oxide donors and L-arginine in experimental stroke; effects on infarct size and cerebral blood flow. Nitric Oxide 12: 141-149, 2005.

14. Paspalj D, Nikic P, Savic M, Djuric D, Simanic I, Zivkovic V, Jeremic N, Srejovic I and Jakovljevic V: Redox status in acute ischemic stroke: Correlation with clinical outcome. Mol Cell Biochem 406: 75-81, 2015.

15. Serrano J, Uttenthal LO, Martínez A, Fernández AP, Martínez de Velasco J, Alonso D, Bentura ML, Santacana M, Gallardo JR, Martínez-Murillo R, et al: Distribution of adrenomedullin-like immunoreactivity in the rat central nervous system by light and electron microscopy. Brain Res 853: 245-268, 2000.

16. López J and Martínez A: Cell and molecular biology of the multifunctional peptide, adrenomedullin. Int Rev Cytol 221: 1-92, 2002.

17. Garayoa M, Martínez A, Lee S, Pío R, An WG, Neckers L, Trepel J, Montuenga LM, Ryan H, Johnson R, et al: Hypoxia-inducible factor-1 (HIF-1) up-regulates adrenomedullin expression in human tumor cell lines during oxygen deprivation: A possible promotion mechanism of carcinogenesis. Mol Endocrinol 14: 848-862, 2000.

18. Serrano J, Alonso D, Encinas JM, Lopez JC, Fernandez AP, Castro-Blanco S, Fernández-Vizarra P, Richart A, Bentura ML, Santacana M, et al: Adrenomedullin expression is up-regulated by ischemia-reperfusion in the cerebral cortex of the adult rat. Neuroscience 109: 717-731, 2002.

19. Serrano J, Fernández AP, Sánchez J, Rodrigo J and Martínez A: Adrenomedullin expression is up-regulated by acute hypobaric hypoxia in the cerebral cortex of the adult rat. Brain Pathol 18: 434-442, 2008

20. Somay G, Halac GU, Uslu E and Aydin S: Plasma adrenomedullin in acute ischemic stroke. Neurosciences (Riyadh) 12: 351-353, 2007.

21. Hurtado O, Serrano J, Sobrado M, Fernández AP, Lizasoain I, Martínez-Murillo R, Moro MA and Martínez A: Lack of adrenomedullin, but not complement factor $\mathrm{H}$, results in larger infarct size and more extensive brain damage in a focal ischemia model. Neuroscience 171: 885-892, 2010.

22. Duangjit S, Muangpaisan W, Chotinaiwattarakul W and Dharmasaroja P: Functional recovery at 3 months in stroke patients not receiving thrombolytic therapy: The comparison between patients arriving earlier and later than $4.5 \mathrm{~h}$. J Stroke Cerebrovasc Dis 23: 91-98, 2014.

23. Berzina G, Sveen U, Paanalahti M and Sunnerhagen KS: Analysing the modified rankin scale using concepts of the international classification of functioning, disability and health. Eur J Phys Rehabil Med, 2015 (Epub ahead of print).

24. Blanco JR, Jarrin I, Martinez A, Siles E, Larrayoz IM, Cañuelo A, Gutierrez F, Gonzalez-Garcia J, Vidal F and Moreno S; CoRIS-Biobanco: Shorter telomere length predicts poorer immunological recovery in virologically suppressed HIV-1-infected patients treated with combined antiretroviral therapy. J Acquir Immune Defic Syndr 68: 21-29, 2015.

25. Martínez A, Elsasser TH, Bhathena SJ, Pío R, Buchanan TA, Macri CJ and Cuttitta F: Is adrenomedullin a causal agent in some cases of type 2 diabetes? Peptides 20: 1471-1478, 1999.

26. Geeganage C, Tracy M, England T, Sare G, Moulin T, Woimant F, Christensen H, De Deyn PP, Leys D, O'Neill D, et al: Relationship between baseline blood pressure parameters (including mean pressure, pulse pressure and variability), and early outcome after stroke: Data from the tinzaparin in acute ischaemic stroke trial (TAIST). Stroke 42: 491-493, 2011.

27. Minnerup J, Wersching H, Unrath M and Berger K: Explaining the decrease of in-hospital mortality from ischemic stroke. PLoS One 10: e0131473, 2015.

28. Pikija S, Trkulja V, Malojcic B, Mutzenbach JS and Sellner J: A high burden of ischemic stroke in regions of Eastern/Central Europe is largely due to modifiable risk factors. Curr Neurovasc Res 12: 341-352, 2015.

29. Morales Vidal SG and Ruland S: Platelet antiaggregants in stroke prevention. Neurol Clin 31: 633-657, 2013.

30. Hiramoto JS, Katz R, Weisman S and Conte M: Gender-specific risk factors for peripheral artery disease in a voluntary screening population. J Am Heart Assoc 3: e000651, 2014.

31. Stroke Unit Trialists' Collaboration: Organised inpatient (stroke unit) care for stroke. Cochrane Database Syst Rev 9: CD000197, 2013.

32. Ishitsuka K, Kamouchi M, Hata J, Fukuda K, Matsuo R, Kuroda J, Ago T, Kuwashiro T, Sugimori H, Nakane H, et al: High blood pressure after acute ischemic stroke is associated with poor clinical outcomes: Fukuoka stroke registry. Hypertension 63: 54-60, 2014. 
33. Tziomalos K, Giampatzis V, Bouziana SD, Spanou M, Papadopoulou M, Kostaki S, Dourliou V, Papagianni M, Savopoulos C and Hatzitolios AI: Elevated diastolic but not systolic blood pressure increases mortality risk in hypertensive but not normotensive patients with acute ischemic stroke. Am J Hypertens 28: 765-771, 2015.

34. Yong M, Diener HC, Kaste M and Mau J: Characteristics of blood pressure profiles as predictors of long-term outcome after acute ischemic stroke. Stroke 36: 2619-2625, 2005.

35. Wohlfahrt P, Krajcoviechova A, Jozifova M, Mayer O, Vanek J, Filipovsky $\mathrm{J}$ and Cifkova R: Low blood pressure during the acute period of ischemic stroke is associated with decreased survival. J Hypertens 33: 339-345, 2015.

36. Vemmos KN, Tsivgoulis G, Spengos K, Zakopoulos N, Synetos A, Manios E, Konstantopoulou P and Mavrikakis M: U-shaped relationship between mortality and admission blood pressure in patients with acute stroke. J Intern Med 255: 257-265, 2004.

37. Bath PM, Martin RH, Palesch Y, Cotton D, Yusuf S, Sacco R, Diener HC, Toni D, Estol C and Roberts R; PRoFESS Study Group: Effect of telmisartan on functional outcome, recurrence and blood pressure in patients with acute mild ischemic stroke: A PRoFESS subgroup analysis. Stroke 40 : 3541-3546, 2009.

38. Potter J, Mistri A, Brodie F, Chernova J, Wilson E, Jagger C, James M, Ford G and Robinson T: Controlling hypertension and hypotension immediately post stroke (CHHIPS)-a randomised controlled trial. Health Technol Assess 13: iii, ix-xi, 2009.

39. Sandset EC, Bath PM, Boysen G, Jatuzis D, Kõrv J, Lüders S, Murray GD, Richter PS, Roine RO, Terént A, et al: The angiotensin-receptor blocker candesartan for treatment of acute stroke (SCAST): A randomised, placebo-controlled, double-blind trial. Lancet 377: 741-750, 2011.

40. Sandset EC, Murray GD, Bath PM, Kjeldsen SE and Berge E; Scandinavian Candesartan Acute Stroke Trial (SCAST) Study Group: Relation between change in blood pressure in acute stroke and risk of early adverse events and poor outcome. Stroke 43: 2108-2114, 2012

41. Bath PM and Krishnan K: Interventions for deliberately altering blood pressure in acute stroke. Cochrane Database Syst Rev 10: CD000039, 2014.

42. Stead LG, Gilmore RM, Vedula KC, Weaver AL, Decker WW and Brown RD Jr: Impact of acute blood pressure variability on ischemic stroke outcome. Neurology 66: 1878-1881, 2006.

43. Fukuda K, Kai H, Kamouchi M, Hata J, Ago T, Nakane H, Imaizumi T and Kitazono T; FSR Investigators; steering committee of the Fukuoka Stroke Registry included: Day-by-day blood pressure variability and functional outcome after acute ischemic stroke: Fukuoka stroke registry. Stroke 46: 1832-1839, 2015

44. Rashid PA, Whitehurst A, Lawson N and Bath PM: Plasma nitric oxide (nitrate/nitrite) levels in acute stroke and their relationship with severity and outcome. J Stroke Cerebrovasc Dis 12: 82-87, 2003.

45. Abdullah A, Ssefer V, Ertugrul U, Osman E, Esref A, Ugur CM, Adalet A, Yavuz Y, Faysal E and Nebahat T: Evaluation of serum oxidant/antioxidant balance in patients with acute stroke. J Pak Med Assoc 63: 590-593, 2013.
46. Cure MC, Tufekci A, Cure E, Kirbas S, Ogullar S, Kirbas A, Unal $\mathrm{H}$, Yuce $\mathrm{S}$ and Cakmak S: Low-density lipoprotein subfraction, carotid artery intima-media thickness, nitric oxide and tumor necrosis factor alpha are associated with newly diagnosed ischemic stroke. Ann Indian Acad Neurol 16: 498-503, 2013.

47. Gonullu H, Aslan M, Karadas S, Kati C, Duran L, Milanlioglu A, Aydin MN and Demir H: Serum prolidase enzyme activity and oxidative stress levels in patients with acute hemorrhagic stroke. Scand J Clin Lab Invest 74: 199-205, 2014.

48. Molnar T, Pusch G, Papp V, Feher G, Szapary L, Biri B, Nagy L, Keki S and Illes Z: The L-arginine pathway in acute ischemic stroke and severe carotid stenosis: Temporal profiles and association with biomarkers and outcome. J Stroke Cerebrovasc Dis 23: 2206-2214, 2014.

49. Salom MG, Arregui B, Carbonell LF, Ruiz F, González-Mora JL and Fenoy FJ: Renal ischemia induces an increase in nitric oxide levels from tissue stores. Am J Physiol Regul Integr Comp Physiol 289: R1459-R1466, 2005.

50. Cui X, Chopp M, Zacharek A, Zhang C, Roberts C and Chen J: Role of endothelial nitric oxide synthetase in arteriogenesis after stroke in mice. Neuroscience 159: 744-750, 2009.

51. Khan M, Sekhon B, Giri S, Jatana M, Gilg AG, Ayasolla K, Elango C, Singh AK and Singh I: S-Nitrosoglutathione reduces inflammation and protects brain against focal cerebral ischemia in a rat model of experimental stroke. J Cereb Blood Flow Metab 25: 177-192, 2005.

52. Zhang H, Tang B, Yin CG, Chen Y, Meng QL, Jiang L, Wang WP and Niu GZ: Plasma adrenomedullin levels are associated with long-term outcomes of acute ischemic stroke. Peptides 52: 44-48, 2014.

53. Xia CF, Yin H, Borlongan CV, Chao J and Chao L: Postischemic infusion of adrenomedullin protects against ischemic stroke by inhibiting apoptosis and promoting angiogenesis. Exp Neurol 197: 521-530, 2006.

54. Wang X, Yue TL, Barone FC, White RF, Clark RK, Willette RN, Sulpizio AC, Aiyar NV, Ruffolo RR Jr and Feuerstein GZ: Discovery of adrenomedullin in rat ischemic cortex and evidence for its role in exacerbating focal brain ischemic damage. Proc Natl Acad Sci USA 92: 11480-11484, 1995.

55. Pedreño M, Morell M, Robledo G, Souza-Moreira L, Forte-Lago I, Caro M, O'Valle F, Ganea D and Gonzalez-Rey E: Adrenomedullin protects from experimental autoimmune encephalomyelitis at multiple levels. Brain Behav Immun 37: 152-163, 2014.

56. Ma S, Zhao H, Ji X and Luo Y: Peripheral to central: Organ interactions in stroke pathophysiology. Exp Neurol 272: 41-49, 2015.

57. Liu J, Yan J, Greer JM, Read SJ, Henderson RD, Rose SE, Coulthard A and McCombe PA: Correlation of adrenomedullin gene expression in peripheral blood leukocytes with severity of ischemic stroke. Int J Neurosci 124: 271-280, 2014.

58. Malfitano AM, Marasco G, Proto MC, Laezza C, Gazzerro P and Bifulco M: Statins in neurological disorders: An overview and update. Pharmacol Res 88: 74-83, 2014.

59. Yamamoto C, Fukuda N, Jumabay M, Saito K, Matsumoto T, Ueno T, Soma M, Matsumoto K and Shimosawa T: Protective effects of statin on cardiac fibrosis and apoptosis in adrenomedullin-knockout mice treated with angiotensin II and high salt loading. Hypertens Res 34: 348-353, 2011. 